\title{
Erratum to: Similar cardiometabolic effects of high- and moderate-intensity training among apparently healthy inactive adults: a randomized clinical trial
}

Robinson Ramírez-Vélez ${ }^{1 *}$ (D) Alejandra Tordecilla-Sanders ${ }^{1}$, Luis Andrés Téllez-T¹ , Diana Camelo-Prieto², Paula Andrea Hernández-Quiñonez ${ }^{1}$, Jorge Enrique Correa-Bautista' ${ }^{1}$ Antonio Garcia-Hermoso³,

Rodrigo Ramirez-Campillo $0^{4,5}$ and Mikel Izquierdo ${ }^{7}$

\section{Erratum to: J Transl Med (2017) 15:118} DOI 10.1186/s12967-017-1216-6

In the original version of this article [1], published on 30 May 2017, we noticed an error in Table 2. The lean mass $(\mathrm{kg})$ and fat mass (\%) corrected table is included in this erratum.
These changes have no material impact on the conclusions of our paper. We apologize for any inconvenience caused to our readers.

*Correspondence: robin640@hotmail.com;

robinson.ramirez@urosario.edu.co

${ }^{1}$ Centro de Estudios para la Medición de la Actividad Física «CEMA»,

Escuela de Medicina y Ciencias de la Salud, Universidad del Rosario,

Bogotá D.C, Colombia

Full list of author information is available at the end of the article 


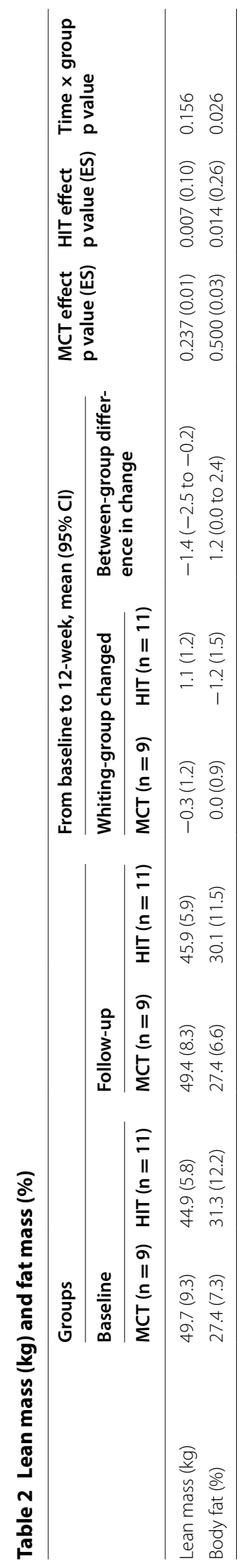




\section{Author details}

${ }^{1}$ Centro de Estudios para la Medición de la Actividad Física «CEMA», Escuela de Medicina y Ciencias de la Salud, Universidad del Rosario, Bogotá D.C, Colombia. ${ }^{2}$ Grupo GICAEDS, Facultad de Cultura Física, Deporte y Recreación, Universidad Santo Tomás, Bogotá D.C, Colombia. ${ }^{3}$ Laboratorio de Ciencias de la Actividad Física, el Deporte y la Salud, Universidad de Santiago de Chile, USACH, Santiago, Chile. ${ }^{4}$ Departamento de Ciencias de la Actividad Física, Universidad de Los Lagos, Osorno, Chile. ${ }^{5}$ Núcleo de Investigación en Salud, Actividad Física y Deporte; Laboratorio de Medición y Evaluación Deportiva, Universidad de Los Lagos, Osorno, Chile. ${ }^{6}$ Unidad de Fisiología Integrativa, Laboratorio del Ciencias del Ejercicio, Clínica MEDS, Santiago, Chile. ${ }^{7}$ Department of Health Sciences, Public University of Navarre, CIBER de Fragilidad y Envejecimiento Saludable (CB16/10/00315), Pamplona, Navarre, Spain.

The online version of the original article can be found under doi:10.1186/s12967-017-1216-6.

\section{Publisher's Note}

Springer Nature remains neutral with regard to jurisdictional claims in published maps and institutional affiliations.

Published online: 13 June 2017

\section{Reference}

1. Ramírez-Vélez R, Tordecilla-Sanders A, Téllez-T LA, Camelo-Prieto D, Hernández-Quiñonez PA, Correa-Bautista JE, Garcia-Hermoso A, RamirezCampillo R, Izquierdo M. Similar cardiometabolic effects of high- and moderate-intensity training among apparently healthy inactive adults: a randomized clinical trial. J Transl Med. 2017:15(1):118. doi:10.1186/ s12967-017-1216-6 